\title{
THE DETERMINISTIC ITÔ-BELATED INTEGRAL IS EQUIVALENT TO THE LEBESGUE INTEGRAL
}

\author{
R. B. DARST AND E. J. MCSHANE
}

\begin{abstract}
Let $[a, b)$ be a bounded half-open interval in the real numbers $R$. Denote by $\mathcal{G}=\mathscr{G}[a, b)$ and $\mathcal{L}=\mathscr{E}[a, b)$ the sets of functions $f: R \rightarrow R$ that are Itô-belated and Lebesgue integrable on $[a, b)$. It is known that $\mathcal{L} \subset \mathcal{G}$, so the assertion in the title is substantiated by showing that $\mathscr{\mathcal { C }} \subset \mathcal{L}$ in the sequel.
\end{abstract}

To set the stage we begin with a definition of the Riemann integral.

A function $f: R \rightarrow R$ is said to be Riemann integrable on $[a, b)$ if there exists a number $J$ such that for each $\varepsilon>0$ there exists $\delta>0$ such that if $\pi=\left\{t_{i}\right\}_{i=0}^{n}$ is a $\gamma$-partition of $[a, b]$ (i.e., $a=t_{0}<t_{1}<\cdots<t_{n}=b$ and $\left.\max _{i}\left(t_{i}-t_{i-1}\right)<\gamma\right)$ and $x_{i} \in\left[t_{i-1}, t_{i}\right]$, then $\left|\left[\sum_{i} f\left(x_{i}\right)\left(t_{i}-t_{i-1}\right)\right]-J\right|<\varepsilon$.

Ralph Henstock and J. Kurzweil [2] independently defined a Riemann-type integral called the Riemann Complete Integral by the following modification of the definition of the integral. Instead of requiring that $\gamma>0$ be a number, they let it be a positive function defined on $R$. Thus, $\pi$ is a $\gamma$-partition if (i) $\max _{i}\left\{\left|x_{i}-t_{i-1}\right|,\left|t_{i}-x_{i}\right|\right\}<\gamma\left(x_{i}\right)$ and (ii) $x_{i} \in\left[t_{i-1}, t_{i}\right]$. Henstock studied this integral and showed that it is more general than the Lebesgue integral. A nice introduction to this theory can be found in [1].

Earlier E. J. McShane and T. A. Botts [3] had defined a Riemann-type integral where the point $x_{i}$ was permitted to fall outside the interval $\left[t_{i-1}, t_{i}\right]$. Introducing this twist to the definition of the integral is accomplished by simply dropping requirement (ii) in the preceding paragraph. This change yields an integral that is equivalent to the Lebesgue integral. A development of the Lebesgue integral by this approach has been completed by E. J. McShane and a book accessible to undergraduates should appear soon.

For stochastic integration, it is appropriate to require that $x_{i} \leqslant t_{i-1}$. Adding this requirement gives types of integrals called belated integrals. Two other natural possibilities arise in the stochastic setting. First, permit $\gamma$ to be zero on a set of Lebesgue measure zero and second, permit sets of intervals with small total measure to be excluded from the averaging of the function. The stochastic integral with these possibilities incorporated, called the Itôbelated integral, has been developed by E. J. McShane (cf. [4], [5]). McShane showed in [4] that the nonbelated deterministic case of this integral is

Received by the editors October 10, 1975.

AMS (MOS) subject classifications (1970). Primary 28A20, 60H05.

Key words and phrases. Itô-belated integral, Lebesgue integral.

(1) American Mathematical Society 1978 
equivalent to the Lebesgue integral. This implies that the deterministic

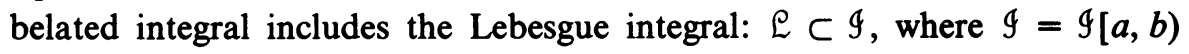
and $\mathcal{E}=\mathcal{E}[a, b)$ denote the sets of functions $f: R \rightarrow R$ that are Itô-belated and Lebesgue integrable on $[a, b)$. However, it has not been known whether they are equivalent or whether, like the Riemann Complete Integral, the belated integral is more general. This note shows that they are equivalent. Before verifying this assertion, it will be convenient to introduce some notation and establish some simple but useful preliminary results.

Let $\gamma: R \rightarrow R$ be nonnegative and positive almost everywhere: $m(\gamma=0)=$ 0 , and let $0<\delta \in R$. Then $\Pi=\left\{\left(A_{i}, x_{i}\right) ; i \leqslant m\right\}$ is a $(\gamma, \delta)$-partition in $[a, b)$ if

(i) $A_{i}=\left[a_{i}, b_{i}\right) \subset[a, b)$,

(ii) $A_{i} \cap A_{j}=\varnothing, i \neq j$,

(iii) $A_{i} \subset\left[x_{i}, x_{i}+\gamma\left(x_{i}\right)\right)$ and

(iv) $m\left([a, b)-\cup A_{i}\right)<\delta$.

Let $\Sigma(f ; \Pi)=\Sigma f\left(x_{i}\right) m\left(A_{i}\right)$. Recall [5, pp. 52-53] that $f \in \mathscr{G}[a, b)$ if, and only if, for each $\varepsilon>0$, there exist $\gamma$ and $\delta$ such that if each of $\Pi_{1}$ and $\Pi_{2}$ is a $(\gamma, \delta)$-partition in $[a, b)$, then $\left|\Sigma\left(f ; \Pi_{1}\right)-\Sigma\left(f ; \Pi_{2}\right)\right|<\varepsilon$; then the pair $(\gamma, \delta)$ is said to correspond to $\varepsilon$ for $f \in \mathscr{9}[a, b)$.

The following facts are verified in [5]:

(1) $9[a, b)$ is a vector space over $R$,

(2) the integral is a linear functional on $\mathscr{G}[a, b)$,

(3) if $[c, d) \subset[a, b)$, then $\mathscr{Y}[a, b) \subset \mathscr{G}[c, d)$, and

(4) if $a \leqslant c \leqslant d \leqslant e \leqslant b$, then $\int_{c}^{d} f+\int_{d}^{e} f=\int_{c}^{e} f$, where $\int_{c}^{d} f$ denotes the Itô-belated integral of $f$ on $[c, d)$.

To show that $G \subset \mathcal{L}$, we will use the preceding facts and the fact that if $f \in \mathscr{G}[a, b)$, then $|f| \in \mathcal{G}[a, b)$; to verify this latter fact the following easy technical lemmas will be handy.

LEMMA 1. Let $(\gamma, \delta)$ correspond to $\varepsilon$ for $f \in \mathscr{G}[a, b)$ and let $\Pi=\left\{\left(A_{i}, x_{i}\right)\right.$; $i \leqslant m\}$ be $a(\gamma, \delta)$-partition in $[a, b)$, where $A_{i}=\left[a_{i}, b_{i}\right)$. Let $S$ be a subset of $Z_{m}=\{1,2, \ldots, m\}$. Then

$$
\left|\sum_{S}\left(f\left(x_{i}\right) m\left(A_{i}\right)-\int_{a_{i}}^{b_{i}} f\right)\right|=\left|\sum_{S} f\left(x_{i}\right) m\left(A_{i}\right)-\sum_{S} \int_{a_{i}}^{b_{i}} f\right| \leqslant \varepsilon .
$$

Proof. Let $\left\{\left[c_{j}, d_{j}\right) ; j \leqslant n\right\}$ be an enumeration of the components of $[a, b)-U_{S} A_{i}$, where $d_{j}<c_{j+1}$. Let $\eta>0$ and let $\left(\gamma_{j}, \delta_{j}\right)$ correspond to $\eta / n$ for $f \in \mathcal{G}\left[c_{j}, d_{j}\right)$. Let $\gamma^{\prime}=\min \left\{\gamma, \gamma_{1}, \ldots, \gamma_{n}\right\}$ and let $\delta^{\prime}=$ $\min \left\{\delta / n, \delta_{1}, \ldots, \delta_{n}\right\}$. Let $\Pi_{j}$ be a $\left(\gamma^{\prime}, \delta^{\prime}\right)$-partition in $\left[c_{j}, d_{j}\right), j \leqslant n$ and let $\Pi^{\prime}$ be the $(\gamma, \delta)$-partition in $[a, b)$ comprised of $\left\{\left(A_{i}, x_{i}\right) ; i \in S\right\} \cup\left\{U_{j} \Pi_{j}\right\}$. Thus 


$$
\begin{aligned}
& \left|\sum_{S} f\left(x_{i}\right) m\left(A_{i}\right)-\sum_{S} \int_{a_{i}}^{b_{i}} f\right| \\
& \quad \leqslant\left|\sum\left(f ; \Pi^{\prime}\right)-\int_{a}^{b} f\right|+\sum_{j}\left|\sum\left(f ; \Pi_{j}\right)-\int_{c_{j}}^{d_{j}} f\right|<\varepsilon+\eta .
\end{aligned}
$$

Corollary 1. Suppose that each of $\Pi_{1}=\left\{\left(A_{i}, x_{i}\right) ; i \leqslant m\right\}$ and $\Pi_{2}=$ $\left\{\left(A_{i}, y_{i}\right) ; i \leqslant m\right\}$ is $a(\gamma, \delta)$-partition in $[a, b)$, where $(\gamma, \delta)$ corresponds to $\varepsilon$ for $f \in \mathscr{9}[a, b)$. Then

(a) $\Sigma\left|f\left(x_{i}\right)-f\left(y_{i}\right)\right| m\left(A_{i}\right) \leqslant 4 \varepsilon$, and

(b) $\left|\Sigma\left(|f| ; \Pi_{1}\right)-\Sigma\left(|f| ; \Pi_{2}\right)\right|<4 \varepsilon$.

Proof. Let $S_{1}=\left\{i ; f\left(x_{i}\right)>f\left(y_{i}\right)\right\}$ and let $S_{2}=[1,2, \ldots, m]-S_{1}$. Then

$$
\begin{aligned}
& \sum_{S_{1}}\left(f\left(x_{i}\right)-f\left(y_{i}\right)\right) m\left(A_{i}\right) \\
& \quad=\sum_{S_{1}}\left\{\left[f\left(x_{i}\right) m\left(A_{i}\right)-\int_{a_{i}}^{b_{i}} f\right]+\left[\int_{a_{i}}^{b_{i}} f-f\left(y_{i}\right) m\left(A_{i}\right)\right]\right\} \leqslant 2 \varepsilon .
\end{aligned}
$$

Similarly $\Sigma_{S_{2}}\left(f\left(y_{i}\right)-f\left(x_{i}\right)\right) m\left(A_{i}\right) \leqslant 2 \varepsilon$, so (a) is established. To establish (b), merely notice that

$$
\begin{aligned}
\left|\sum\right| f\left(x_{i}\right)\left|m\left(A_{i}\right)-\sum\right| f\left(y_{i}\right)\left|m\left(A_{i}\right)\right| & =\left|\sum\left(\left|f\left(x_{i}\right)\right|-\left|f\left(y_{i}\right)\right|\right) m\left(A_{i}\right)\right| \\
& \leqslant \sum\left|\left(\left|f\left(x_{i}\right)\right|-\left|f\left(y_{i}\right)\right|\right)\right| m\left(A_{i}\right) \\
& \leqslant \sum\left|f\left(x_{i}\right)-f\left(y_{i}\right)\right| m\left(A_{i}\right) .
\end{aligned}
$$

LEMMA 2. Let $\Pi=\left\{\left(A_{i}, x_{i}\right) ; i \leqslant m\right\}$ be $a(\gamma, \delta)$-partition in $[a, b)$, where $(\gamma, \delta)$ corresponds to $\varepsilon$ for $f \in \mathscr{I}[a, b)$. Let $\left\{B_{j} ; j<n\right\}$ be a finite collection of pairwise disjoint half-open intervals $\left[c_{j}, d_{j}\right)$ in $[a, b)-A$, where $A=\cup A_{i}$. Let $\Pi_{1}=\left\{\left(B_{j}, y_{j}\right) ; j \leqslant n\right\}$ and suppose that $\Pi_{2}=\Pi \cup \Pi_{1}$ is $a(\gamma, \delta)$-partition in $[a, b)$. Then

(a) $\left|\Sigma\left(f ; \Pi_{1}\right)\right|<2 \varepsilon$, and

(b) $\Sigma\left(|f| ; \Pi_{1}\right)<4 \varepsilon$.

Proof. To obtain (a) notice that $\left|\Sigma\left(f ; \Pi_{1}\right)\right|=\left|\Sigma\left(f ; \Pi_{2}\right)-\Sigma(f ; \Pi)\right|<2 \varepsilon$; then apply (a) to the positive and negative terms separately and verify (b).

Lemma 3. Let $f \in \mathscr{G}[a, b)$; then $|f| \in \mathscr{G}[a, b)$.

Proof. Let $\varepsilon>0$ and choose $(\gamma, \delta)$ such that if each of $\Pi^{1}$ and $\Pi^{2}$ is a $(\gamma, 2 \delta)$-partition in $[a, b)$, then $\left|\Sigma\left(f ; \Pi^{1}\right)-\Sigma\left(f ; \Pi^{2}\right)\right|<\varepsilon / 12$. Let each of $\Pi_{1}=\left\{\left(A_{i}, x_{i}\right) ; i \leqslant m\right\}$ and $\Pi_{2}=\left\{\left(B_{j}, y_{j}\right) ; j \leqslant n\right\}$ be a $(\gamma, \delta)$-partition in $[a, b)$. Let $\Pi_{3}=\left\{\left(A_{i} \cap B_{j}, x_{i}\right) ; i \leqslant m, j \leqslant n\right\}, \Pi_{4}=\left\{\left(A_{i} \cap B_{j}, y_{j}\right) ; i \leqslant m\right.$, $j \leqslant n\}, A=\cup A_{i}$ and $B=\cup B_{j}$. Then let $\Pi_{5}$ correspond to the components of $A_{i}-B$ taken with $x_{i}$, where $i \leqslant m$, and let $\Pi_{6}$ correspond to the components of $B_{j}-A$, taken with $y_{j}$, where $j \leqslant n$. Thus 


$$
\begin{aligned}
& \sum\left(|f| ; \Pi_{1}\right)=\sum\left(|f| ; \Pi_{3}\right)+\sum\left(|f| ; \Pi_{5}\right), \quad \text { and } \\
& \sum\left(|f| ; \Pi_{2}\right)=\sum\left(|f| ; \Pi_{4}\right)+\sum\left(|f| ; \Pi_{6}\right) .
\end{aligned}
$$

Lemma 2(b) implies that $\Sigma\left(|f| ; \Pi_{5}\right)<4(\varepsilon / 12)$ and $\Sigma\left(|f| ; \Pi_{6}\right)<4(\varepsilon / 12)$, and Corollary $1(\mathrm{~b})$ asserts that $\left|\Sigma\left(|f| ; \Pi_{3}\right)-\Sigma\left(|f| ; \Pi_{4}\right)\right| \leqslant 4(\varepsilon / 12)$. Thus

$$
\begin{aligned}
& \left|\sum\left(|f| ; \Pi_{1}\right)-\sum\left(|f| ; \Pi_{2}\right)\right| \\
& \quad \leqslant\left|\sum\left(|f| ; \Pi_{3}\right)-\sum\left(|f| ; \Pi_{4}\right)\right|+\sum\left(|f| ; \Pi_{5}\right)+\sum\left(|f| ; \Pi_{6}\right)<\varepsilon .
\end{aligned}
$$

Having established Lemma 3, we observe that if $f$ is in $9[a, b)$, then each of $f \vee 0=(f+|f|) / 2$ and $f \wedge 0=(f-|f|) / 2$ is also in $9[a, b)$; thus, to verify that $\mathcal{L} \subset \mathcal{G}$, we suppose that $0 \leqslant f \in(G-\mathcal{L})$ and therefrom obtain a contradiction as follows.

First, we notice that if $f \wedge n$ were in $\mathcal{L}$ for every positive integer $n$, then the inequalities (L) $\int_{a}^{b}(f \wedge n)=\int_{a}^{b}(f \wedge n) \leqslant \int_{a}^{b} f<\infty$ would be valid and $f$ would be in $\mathcal{L}$, where (L) denotes the Lebesgue integral. Thus we also suppose that $0 \leqslant f \leqslant n$ which implies that $0 \leqslant \int_{s}^{t} f \leqslant n(t-s), a \leqslant s \leqslant t \leqslant$ $b$. Hence the equation $F(t)=\int_{a}^{t} f$ defines a Lipschitz function on $[a, b]$, and there exists $g \in \mathcal{L}$ with $0 \leqslant g \leqslant n$ and $F(t)=(\mathrm{L}) \int_{a}^{t} g=\int_{a}^{t} g, a \leqslant t \leqslant b$. Denote $f-g$ by $h$. Then $h \in \mathcal{G}-\mathcal{L},|h| \leqslant n$ and $\int_{s}^{t} h=0, a \leqslant s \leqslant t \leqslant b$. Since $h \notin \mathcal{L}$, there exists $\varepsilon>0$ such that $m^{*}(|h|>\varepsilon)>8 \varepsilon$. Multiplying by -1 and relabeling if necessary, suppose that $m^{*}(h>\varepsilon)>4 \varepsilon$. Remembering that $h \in \mathcal{G}$, choose $(\gamma, \delta)$ corresponding to $\varepsilon^{2}$ for $h \in \mathcal{G}=\mathscr{9}[a, b)$. Denote $(h>\varepsilon) \cap(\gamma>0)$ by $E$. From the Vitali covering $\{[x, x+\alpha] ; x \in E, 0<\alpha$ $<\gamma(x)\}$ of $E$ choose a finite sequence of pairwise disjoint elements $\left\{\left[x_{i}, x_{i}+\right.\right.$ $\alpha_{i}$ ]; $\left.i \leqslant m\right\}$ with $x_{i}<x_{i+1}$ and $\sum \alpha_{i}>3 \varepsilon$. On each complementary half-open interval, $I_{0}=\left[a, x_{1}\right), \quad I_{j}=\left[x_{j}+\alpha_{j}, x_{j+1}\right), \quad I_{m}=\left[x_{m}+\alpha_{m}, b\right)$, choose a $(\gamma, \delta /(m+1))$-partition $\Pi_{j}$ such that $\left|\Sigma\left(h ; \Pi_{j}\right)\right|<\varepsilon^{2} /(m+1)$. Let $\Pi$ be the $(\gamma, \delta)$-partition generated by the elements $\left(\left[x_{i}, x_{i}+\alpha_{i}\right), x_{i}\right), 1 \leqslant i \leqslant n$ and the elements of $\Pi_{j}, 0 \leqslant j \leqslant m$. Thus, the contradiction

$$
\varepsilon^{2}>\left|\sum(h ; \Pi)\right|>\varepsilon \sum \alpha_{i}-\sum_{j}\left|\sum\left(h ; \Pi_{j}\right)\right|>3 \varepsilon^{2}-\varepsilon^{2}
$$

obtains.

We mention that if $g:[a, b) \rightarrow R$ is of bounded variation on $[a, b)$, then one can show that the Lebesgue-Stieltjes integral (LS) $\int_{a}^{b} f d g$ of $f$ with respect to $g$ on $[a, b)$ exists if, and only if, the corresponding Itô-belated integral (I) $\int_{a}^{b} f d g$ exists. To verify this latter assertion, recall that $g$ splits uniquely into a jump function and the difference of two mutually singular, continuous, nondecreasing functions, so the verification reduces to considering the case where $g$ is continuous and nondecreasing. Then use a slight twist of Vitali's Theorem to adjust the argument that we gave for the case $g(x)=x$. 


\section{REFERENCES}

1. Ralph Henstock, A Riemann-type integral of Lebesgue power, Canad. J. Math. 20 (1968), 79-87.

2. J. Kurzweil, Generalized ordinary differential equations and continuous dependence on a parameter, Czechoslovak Math. J. 7 (82) (1957), 418-446.

3. E. J. McShane and T. A. Botts, A modified Riemann-Stieltjes integral, Duke Math. J. 19 (1952), 293-302.

4. E. J. McShane, A Riemann-type integral that includes Lebesgue-Stieltjes, Bochner and stochastic integrals, Mem. Amer. Math. Soc., No. 88, 1969.

5. __ Stochastic calculus and stochastic models, Academic Press, New York, 1974.

Department of Mathematics, Colorado State University, fort Collins, Colorado 80523 (Current address of R. B. Darst)

Current address (E. J. McShane): Department of Mathematics, University of Virginia, Charlottesville, Virginia 22903 